\title{
On the Long-Range Attraction between Proteins Due to Nonadsorbing Polysaccharide
}

\author{
R. Tuinier ${ }^{*},+, \neq$ and A. Brület§ \\ Van 't Hoff Laboratory, Debye Research Institute, University of Utrecht, Padualaan 8, \\ $3584 \mathrm{CH}$ Utrecht, The Netherlands, Forschungszentrum Jülich, Institut für Festkörperforschung, \\ Weiche Materie, D-52425 Jülich, Germany, and Laboratoire Leon Brillouin (CEA-CNRS), CE-Saclay, \\ 91191 Gif-Sur-Yvette Cedex, France
}

Received April 18, 2002; Revised Manuscript Received August 23, 2002

\begin{abstract}
The effect of a nonadsorbing polysaccharide (dextran) on the structure factor of a solution of lysozyme was studied using small-angle neutron scattering (SANS) experiments. By choosing the appropriate water/ deuterium ratio as solvent, we made the scattering signal from dextran invisible for the SANS measurements. Dextran induces a weak long-range attraction between the lysozyme molecules. This attraction is described using a depletion interaction potential from theory for two spheres in an ideal polymer solution. Incorporation of the theory in a mean-spherical approximation shows that the wave vector below which the structure factor increases depends on the polymer size. The theoretical prediction is in fair agreement with the measured structure factor of lysozyme, as affected by nonadsorbing dextran.
\end{abstract}

\section{Introduction}

Since the pioneering work of de Gennes, ${ }^{1}$ depletion type of interaction between proteins induced by nonadsorbing long polymer chains has received much theoretical ${ }^{2-7}$ attention. A solution of proteins immersed in a semidilute polymer solution was described by de Gennes as a mixture of small colloidal spheres (the proteins) having a radius, $R$, immersed in a polymer solution with a characteristic correlation length, $\xi$. De Gennes ${ }^{1}$ showed that the depletion-induced attraction is strong when $R>\xi$ but becomes very weak when $R<\xi$. Most polysaccharide-protein mixtures are examples of $R$ $<\xi$; in their native state, proteins have a diameter of a few nanometers, whereas polysaccharides have a radius of gyration (dilute) or correlation length (semidilute) often one or two magnitudes larger. The stability of polysaccharideprotein mixtures is an important issue because both biopolymers are present in many food products..$^{8,9}$ The properties of polymer-protein mixtures have been intensively studied over the past decade ${ }^{8-11}$ (for a review on mixtures of polysaccharides and proteins, see ref 11). Although also significant theoretical progress was made, a sufficient synergy with experimental work is still lacking.

This study links small-angle neutron scattering (SANS) data to a recent theoretical approach for colloid-polymer mixtures. ${ }^{6}$ As a system to study polysaccharide-induced interactions between proteins, a mixture of lysozyme and dextran was chosen. Lysozyme is a globular protein with a molar mass of $14.400 \mathrm{~kg} / \mathrm{mol}$ and is classically described as an ellipsoid of revolution with a main radius of $2.25 \mathrm{~nm}$

* To whom correspondence should be addressed. Correspondence address: Forschungszentrum Jülich, Institut für Festkörperforschung, Weiche Materie, D-52425 Jülich, Germany. Electronic mail: r.tuinier@fz-juelich.de.

$\dagger$ University of Utrecht.

$\doteqdot$ Institut für Festkörperforschun.

$\S$ Laboratoire Leon Brillouin (CEA-CNRS). and an aspect ratio of $1.5,{ }^{12}$ giving an effective radius of gyration close to $R_{\mathrm{g}}=2 \mathrm{~nm}$. Using the specific volume of lysozyme of $0.702 \pm 0.003 \mathrm{~L} / \mathrm{kg}$ at $25^{\circ} \mathrm{C},{ }^{13}$ together with the molar mass of lysozyme of $14.4 \mathrm{~kg} / \mathrm{mol}$, we estimated the volume per lysozyme as $1.7 \times 10^{-26} \mathrm{~m}^{3}$. If this volume is set equal to an effective sphere of volume, $4 \pi R_{\mathrm{eff}} 3 / 3$, the effective sphere radius, $R_{\text {eff }}$, equals $1.6 \mathrm{~nm}$. This is in fair agreement with small-angle X-ray scattering (SAXS) of Castelletto et al., ${ }^{14}$ who could describe form factor $P(Q)$ at small wave vector $Q$ with the Guinier approximation, $P(Q)$ $=\exp \left(-Q^{2} R_{\mathrm{g}}{ }^{2} / 3\right)$, with a radius of gyration of $1.5 \mathrm{~nm}$. If lysozyme is considered as a hard sphere, its radius equals $R_{\mathrm{g}} \sqrt{ }(5 / 3)=2.0 \mathrm{~nm}$. From the calculated pair distribution function derived from the SAXS data, Castelletto et al. ${ }^{14}$ could also determine a largest diameter of the molecule of $5.0 \mathrm{~nm}$. The effective hydrodynamic radius is $2.1 \mathrm{~nm} .{ }^{15}$ Lysozyme can thus be fairly approximated as a sphere. Lysozyme has the advantage that it has been studied intensely; it easily crystallizes in aqueous salt solutions. ${ }^{16-21}$ The effect of concentration or type of salt on the phase behavior of lysozyme solutions are interesting aspects of protein crystallography. ${ }^{16}$ The isoelectric $\mathrm{pH}$ of lysozyme is 11.2. ${ }^{16}$ The experiments reported in this communication were made in $0.10 \mathrm{M}$ phosphate buffer at $\mathrm{pH} 6.0$ in which lysozyme molecules have a net charge of approximately 9.0. ${ }^{16}$ At this $\mathrm{pH}$, lysozyme is soluble and crystallization only occurs at high protein volume fractions, $\phi$. Recently, Poon et al. ${ }^{17}$ showed that a universal (i.e., independent of the salt type) fluid-crystal phase boundary is observed if the ratio of the salt concentration over the net lysozyme charge is plotted as a function of the protein volume fraction. Following the universal crystallization boundary of Poon et al., ${ }^{17}$ crystallization is expected above $\phi=0.1$ under the conditions in this study. At a ionic strength of $0.10 \mathrm{M}$, the protein is expected to be well-screened but not screened to such a 
degree that van der Waals and possible other types of attraction dominate. This happens at higher ionic strengths at which crystallization is enhanced. Actually, at a ionic strength of $0.10 \mathrm{M}$, Gripon et al. ${ }^{22}$ measured a positive second osmotic virial coefficient for lysozyme close to $3.2 \times 10^{-4}$ mol cm $\mathrm{cm}^{3}$ at $27^{\circ} \mathrm{C}$. This virial coefficient corresponds to an effective excluded volume of about six times the volume of a lysozyme molecule. Under the conditions chosen in our experiment, it is reasonable to adduce that lysozyme behaves effectively as a hard sphere. At $25^{\circ} \mathrm{C}$, a solubility limit of lysozyme was found at $230 \mathrm{~g} / \mathrm{L}$, corresponding to $\phi=0.16$, above which crystallization occurs. The aim here is to measure the effect on the interactions between the lysozyme molecules due to dextran, which, to our knowledge, has not been done earlier. SANS enables a measurement of the partial structure factors of the individual scattering components by choosing suitable labeling conditions. In the next section, how the structure factor is computed from depletion interaction theory is explained.

\section{Theory}

The structure factor can be calculated when $c(Q)$, the Fourier transform of the direct correlation function $c(r)$, where $r$ is the distance between the centers of the colloidal spheres with radius $R$, is known: ${ }^{23}$

$$
S(Q)=\left(1-\frac{3 \phi c(Q)}{4 \pi R^{3}}\right)^{-1}
$$

Here $c(r)$ is calculated using the mean spherical approximation (MSA), which is a perturbation of the Percus-Yevick closure relation. The MSA expression for $c(r)$ reads as follows:

$$
c(r)= \begin{cases}c_{\mathrm{HS}}(r) & r \leq 2 R \\ W(r) /(k T) & r>2 R\end{cases}
$$

where $c_{\mathrm{HS}}(r)$ is the Percus-Yevick ${ }^{24}$ direct correlation function for hard spheres, which is accurate at least up to an order above the volume fractions relevant for this study, and $W(r)$ is the depletion interaction potential due to the presence of nonadsorbing polymers. The quantities $k$ and $T$ have their usual meaning as Boltzmann's constant and temperature, respectively.

The interaction potential $W(r)$ between two colloidal spheres in a solution with nonadsorbing polymers can be calculated from the polymer density profiles in the space surrounding the two spheres. An analytical expression for the segment density profile of ideal polymers around a single sphere is available. ${ }^{25,2}$ It is especially important for relatively small spheres compared to the polymers that polymer packing effects around the sphere are taken into account rather than just considering their radius of gyration as effective depletion layer thickness. By comparison with computer simulation results, it was established that the segment concentration around two interacting spheres is described very well by a simple product of the polymer density profiles around a single sphere for any colloid-polymer size ratio. ${ }^{6}$ Thus, the polymer density profiles around the two spheres, obtained

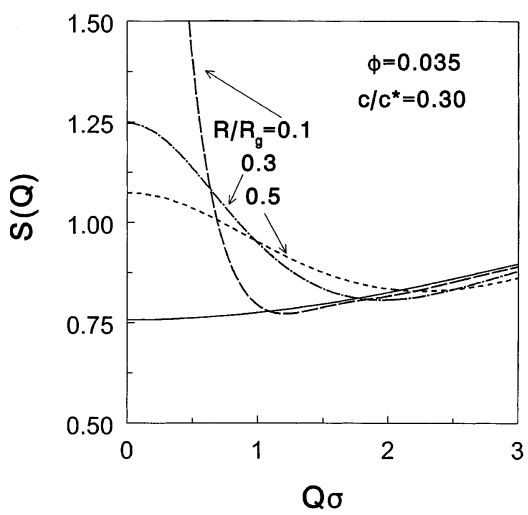

Figure 1. Effect of the polymer-colloid size ratio on the structure factor $S(Q)$ (dashed curves for $R / R_{\mathrm{g}}=0.1,0.3$, and 0.5 , as indicated) for small colloids, as compared to the hard sphere structure factor (full curve). The polymer concentration is $c / c^{*}=0.3$, and the colloid volume fraction is $3.5 \mathrm{vol} \%$.

from the single sphere density profiles, allow a calculation of the interaction potential. ${ }^{6}$

In Figure 1, the structure factor $S(Q)$ is plotted as a function of $Q \sigma$ (where $\sigma=2 R$ ) for three colloid-polymer size ratios, $R / R_{\mathrm{g}}=0.1,0.3$, and 0.5 , for a volume fraction of the colloids of 0.035 (which is the lysozyme volume fraction in this study) as dashed curves for $c / c^{*}=0.3$, where $c$ is the polymer concentration and $c^{*}$ is the overlap concentration. The full curve is the hard sphere result. It is obvious that the attractions lead to an upswing of $S(Q)$ at small $Q$. As the relative size of the polymer becomes larger, the wave vector at which $S(Q)$ increases becomes smaller; longer-range attractions are probed at smaller wave vectors, as was shown first with scaling theory by Sear. ${ }^{26}$ The increase of $S(Q)$ at small wave vectors is stronger as the relative polymer size increases.

\section{Materials and Methods}

The lysozyme and dextran were purchased from Sigma and Fluka, respectively. We mixed aqueous solutions of lysozyme with solutions of dextran. Dextran is a polysaccharide that can be made by the bacterium Leuconostoc mesenteroides. The backbone of this polysaccharide mainly consists of $\alpha(1 \rightarrow 6)$-linked glucoses. ${ }^{27}$ The dextran sample has a number-averaged radius of gyration $\left(R_{\mathrm{g}}\right)$ in aqueous solution of $20 \pm 1 \mathrm{~nm}$, and number- and weight-averaged molar masses of $M_{\mathrm{n}}=260$ and $M_{\mathrm{w}}=387 \mathrm{~kg} / \mathrm{mol}$, respectively, as determined using a combination of size exclusion chromatography and static light scattering at NIZO food research in Ede, The Netherlands, described in ref 28. For dextran, a Flory-Huggins parameter of 0.48 is reported in aqueous salt solutions, ${ }^{29}$ indicating that it can nearly be described with Gaussian statistics. The overlap concentration, here defined as $c^{*}=3 M /\left(N_{\mathrm{av}} 4 \pi R_{\mathrm{g}}{ }^{3}\right)$, is $13 \pm 3 \mathrm{~g} / \mathrm{L}$ if the number-averaged molar mass is used and $19 \pm 4 \mathrm{~g} / \mathrm{L}$ for the weight-averaged molar mass.

SANS measurements were performed with the PAXE spectrometer (Saclay, France) of the Laboratory Léon Brillouin at the Orphée reactor. The sample detector distance was $3.0 \mathrm{~m}$, and the neutron wavelength was $0.84 \mathrm{~nm}$ with a wavelength spread of $10 \%$. By choosing appropriate mixtures 
of deuterated and protonated solvent $\left(\mathrm{D}_{2} \mathrm{O} / \mathrm{H}_{2} \mathrm{O}\right)$, we were able to measure the partial structure factors of dextran and lysozyme separately. Polysaccharide can be made invisible in aqueous solution by choosing the appropriate $\mathrm{H}_{2} \mathrm{O} / \mathrm{D}_{2} \mathrm{O}$ ratio, which is characterized by where $x_{\mathrm{D}}$, the fraction of $\mathrm{D}_{2} \mathrm{O}$. Because dextran mainly consists of glucose, which in polymerized form has a molar mass of $162 \mathrm{~g} / \mathrm{mol}$, the scattering length density is estimated as $1.17 \times 10^{10} \mathrm{~cm}^{-2}$. $\mathrm{H}_{2} \mathrm{O}$ has a scattering length density of $-0.56 \times 10^{10} \mathrm{~cm}^{-2}$ and $\mathrm{D}_{2} \mathrm{O} 6.39 \times 10^{10} \mathrm{~cm}^{-2}$. Thus, the dextran signal can be matched with a solvent having $x_{\mathrm{D}}=0.25$. For lysozyme, Niimura et al. ${ }^{30}$ made SANS contrast variation measurements from which it followed that lysozyme is matched at $x_{\mathrm{D}}=$ 0.43 , which is a common value for proteins. ${ }^{31}$ At the matching condition for dextran $\left(x_{\mathrm{D}}=0.25\right)$, the contrast for lysozyme is still significant, although the scattering intensity can be estimated to be reduced by a factor of 6 compared to using pure $\mathrm{D}_{2} \mathrm{O}$. For the experiments reported here, it was first checked whether the matching points were indeed at $x_{\mathrm{D}}$ $=0.25$ for dextran and at $x_{\mathrm{D}}=0.43$ for lysozyme. Dilute dextran solutions were prepared of $4 \mathrm{~g} / \mathrm{L}$ in $0.10 \mathrm{M}$ phosphate buffer at $\mathrm{pH} 6.0$ (the $\mathrm{pH}$ as measured with a $\mathrm{pH}$ meter was adjusted to $\mathrm{pH} 6.0$ with $\mathrm{NaOH}$ and $\mathrm{HCl}$ ) with $x_{\mathrm{D}}=0,0.20$, 0.80 , and 1.00. Similar solvent mixtures were used to dilute lysozyme to a concentration of $50 \mathrm{~g} / \mathrm{L}(\phi=0.035)$. The match points were determined from the condition $I(Q) \rightarrow 0$, as derived from a plot of the root of the scattering intensity versus $x_{\mathrm{D}}$. The match points corresponded to $x_{\mathrm{D}}=0.42 \pm$ 0.02 for lysozyme and $x_{\mathrm{D}}=0.25 \pm 0.02$ for dextran. Thus, suspensions with $x_{\mathrm{D}}=0.25$ were prepared of solvent and lysozyme $(\phi=0.035)$ mixed with dextran, and pure lysozyme $(\phi=0.035)$ as reference system. Because of the small contrast, the measuring time was $8 \mathrm{~h}$ per sample to reduce the statistical errors to an acceptable level. Measurements at the matching condition for lysozyme were made as well, but the statistical errors were too large for a proper interpretation. The data were corrected for background and empty cell via standard procedures.

\section{Results and Discussion}

Phase Behavior. Lysozyme was mixed with dextran at an ionic strength of $0.10 \mathrm{M}$ (phosphate buffer) at $\mathrm{pH}$ 6.0. Because dextran is a neutral polysaccharide, it does not adsorb onto lysozyme; only oppositely charged polysaccharides and proteins tend to associate. ${ }^{9,11}$ The phase boundary of lysozyme/dextran mixtures in $0.10 \mathrm{M}$ phosphate buffer at $\mathrm{pH} 6.0$ at $25^{\circ} \mathrm{C}$ from visual observation is plotted in Figure 2. Stable mixtures remained fully transparent upon mixing dextran and lysozyme. A clear upper phase and a turbid lower phase could identify the unstable region. The turbid phase sometimes appeared somewhat gellike. It is clear that the mixture is stable up to quite high dextran concentrations with respect to the overlap concentration. Below a lysozyme volume fraction of 0.16 , no crystallization is found upon adding dextran. Probably the dextran sample is too large to effectively introduce a short-range attraction.

Phase separation that occurs at very high dextran concentration can be explained by a depletion-induced attraction

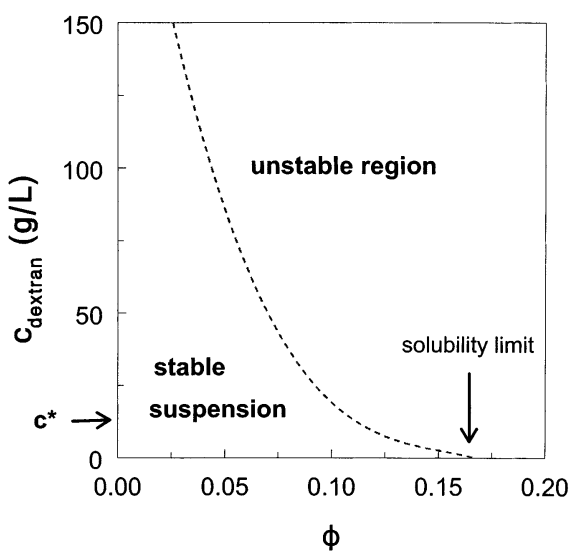

Figure 2. Phase diagram of lysozyme and dextran in $0.10 \mathrm{M}$ phosphate buffer at $\mathrm{pH}$ 6.0. Below the drawn curve, the systems are stable, and above the curve, a phase separation occurs as judged by visual observation.

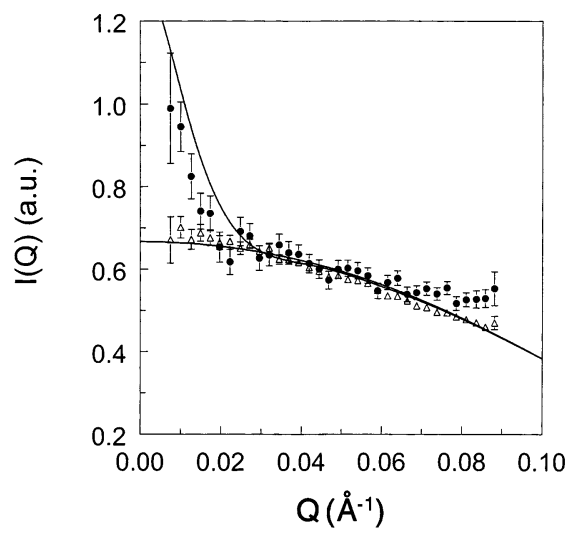

Figure 3. Small-angle neutron scattering intensity of a $3.5 \mathrm{vol} \%$ lysozyme dispersion $(\Delta)$ and of a $3.5 \mathrm{vol} \%$ lysozyme $/ 4.0 \mathrm{~g} / \mathrm{L}$ dextran mixture $(\bullet)$ at $x_{D}=0.25$. The full curves are results of the theoretical models described in the text for $c / c^{*}=0.2$ and a colloid volume fraction of $3.5 \mathrm{vol} \%$ for $R_{\mathrm{g}} / R=8$.

between the lysozyme molecules due to the nonadsorbing dextran. It is therefore intriguing to try to measure such attractions, which is possible by using SANS.

Small-Angle Neutron Scattering (SANS). The (corrected) measured scattering intensity of the pure lysozyme solution at $x_{\mathrm{D}}=0.25$ is plotted as a function of the wave vector $Q$ in Figure $3(\triangle)$. The solid curve (the lower curve at small $Q$ ) shows the theoretical result for lysozyme in solution calculated by $I(Q)=$ const $\times S_{\mathrm{HS}}(Q) P(Q)$, where $S_{\mathrm{HS}}(Q)$ is the Percus-Yevick hard sphere structure factor and with the Guinier approximation $P(Q)=\exp \left(-\left[Q^{2} R_{\mathrm{g}}{ }^{2} /\right.\right.$ 3]) with $R_{\mathrm{g}}=1.5 \mathrm{~nm}(R=2.0 \mathrm{~nm})$, as is in agreement with the results of Castelletto et al. ${ }^{14}$ The constant was fitted such that the curve is shifted best toward the experimental data.

The results for a mixture of lysozyme with (invisible) 4 $\mathrm{g} / \mathrm{L}$ dextran (at $x_{\mathrm{D}}=0.25$ ) are also plotted in Figure 3. For this mixture, it is noted that we are far below the phaseseparation threshold, see Figure 2. It follows from Figure 3 that the results (with and without dextran) are very comparable for $Q>0.03 \AA$, except for $Q>0.07 \AA$ at which the scattering intensity for the mixture seems to be somewhat larger. This could be an effect of the larger statistical errors for $Q>0.07 \AA$. The main difference is found at small $Q$. The results are described as $I(Q)=$ const $\times S(Q) P(Q)$, where the shifting constant and $P(Q)$, the form factor of lysozyme, 
were kept the same as before. The effective structure factor of the lysozyme solution with invisible dextran is described as follows. The depletion interaction potential that perturbs the hard sphere structure factor is calculated under the assumption that dextran can be described as a quasi-ideal polymer. Odijk ${ }^{32}$ showed that in fairly good solvents the polymers can be regarded as quasi-ideal for relatively small colloidal spheres. This supposition can be justified by the fact that the length scale over which small spheres interact with the polymer chains is small compared to the coil size. Further the solvent quality of aqueous dextran solutions can be described by a Flory-Huggins parameter just below $1 / 2$. Therefore, with respect to the effective depletion interaction induced between the lysozyme molecules as imposed by dextran, the interaction potential was calculated from a model for two hard spheres in an ideal polymer solution for arbitrary colloid-polymer size ratio, ${ }^{6}$ as described in the theoretical section. In the calculations of the depletion potential $W(r)$, lysozyme was treated as a hard sphere with an interaction radius of $2.5 \mathrm{~nm}$, so $R_{\mathrm{g}} / R=8$.

A reasonable description of the experimental data could be obtained when using a concentration of polymers of $c / c^{*}$ $=0.2$ in the calculations. This is not unreasonable because the dextran concentration is $4 \mathrm{~g} / \mathrm{L}$ and the (weight-averaged) overlap concentration is $19 \mathrm{~g} / \mathrm{L}$. The theoretical prediction obtained in this way (at low $Q$, upper curve in Figure 3) gives a fair description of the experimental results. The longrange attraction between the lysozyme molecules results in an increase in the scattering intensity at very small $Q$. The finding that long-range attraction between colloidal spheres would be measurable at small wave vectors was already predicted by Sear ${ }^{26}$ using scaling theory. Our experimental data confirm this for a biopolymer mixture. We have an indication therefore that SANS is a promising method to directly measure the long-range interaction potential between proteins in solutions with nonadsorbing polysaccharides. In the future, such work might be linked to crystallization to resolve the issue of the influence on the range of the attractive potential on protein crystallization.

\section{Conclusions}

Small-angle neutron (SANS) measurements have been made to study the attractions induced by a nonadsorbing dextran between lysozyme molecules. The scattering intensity of a lysozyme dispersion mixed with dextran, which was made invisible for neutrons by matching the solvent, was measured. A significant increase of the structure factor at small wave vectors was observed when dextran was added to the lysozyme dispersion. The observed effect on the structure factor could be described well with a theory for the interaction potential between small colloids in the presence of large nonadsorbing polymers.

Acknowledgment. These investigations were supported by the Council for Chemical Sciences of The Netherlands Organization for Scientific Research (NWO/CW) and Unilever Research Vlaardingen. R.T. expresses his thanks to J.
Suurmond for technical assistance and M. Ramzi for sharing the beam time in Saclay. S. Rathgeber and J.E. O'Connell are acknowledged for a critical reading of the manuscript. Useful discussions with M. Fuchs, J. Drenth, G. A. Vliegenthart, A. Vrij, C. G. de Kruif, and H. N. W. Lekkerkerker have contributed to an understanding and interpretation of the measurements. G. A. Vliegenthart, J. K. G. Dhont, and an anonymous reviewer are thanked for noting an error in the $S(Q)$ calculations in an earlier version of the manuscript.

\section{References and Notes}

(1) De Gennes, P. G. C. R. Acad. Sci. B 1979, $288,359$.

(2) Eisenriegler, E.; Hanke, A.; Dietrich, S. Phys. Rev. E 1996, 54, 1134.

(3) Odijk, T. Physica A 2000, 278, 347

(4) Sear, R. P. Phys. Rev. E 1998, 58, 724; J. Chem. Phys. 2001, 115 575; Phys. Rev. Lett. 2001, 86, 4696.

(5) Fuchs, M.; Schweizer, K. S. Europhys. Lett. 2000, 51, 621; Phys. Rev. E 2001, 64, 021514.

(6) Tuinier, R.; Vliegenthart, G. A.; Lekkerkerker, H. N. W. J. Chem. Phys. 2000, 113, 10768.

(7) Eisenriegler, E. J. Phys.: Condens. Matter 2000, 12, A227; J. Chem. Phys. 2000, 113, 5091.

(8) Grinberg, V. Ya.; Tolstoguzov, V. B. Food Hydrocolloids 1997, 11, 145.

(9) Syrbe, A.; Bauer, W. J.; Klostermeyer, H. Int. Dairy J. 1998, 8, 179.

(10) Kulkarni, A. M.; Chatterjee, A. P.; Schweizer, K. S.; Zukoski, C. F. Phys. Rev. Lett. 1999, 83, 4554.

(11) Doublier, J.-L.; Garnier, C.; Renard, C.; Sanchez, C. Curr. Opin. Colloid Interface Sci. 2000, 5, 184.

(12) Tanford, C.; Wagner, M. L. J. Am. Chem. Soc. 1954, 76, 3331.

(13) Chalikian, T. V.; Totrov, M.; Abagyan, R.; Breslauer, K. J. J. Mol. Biol. 1996, 260, 588.

(14) Castelletto, V.; Arêas, E. P. G.; Arêas, J. A. G.; Craievich, A. F. J. Chem. Phys. 1998, 109, 6133.

(15) Eberstein, W.; Georgalis, Y.; Saenger, W. J. Cryst. Growth 1994, $143,71$.

(16) Weber, P. C. In Macromolecular Crystallography. Part A; Carter, C. W., Jr., Sweet, R. M., Eds.; Methods in Enzymology, Vol. 276; Academic Press: San Diego, CA, 1997; Chapter 2, pp 36 and 37.

(17) Poon, W. C. K.; Egelhaaf, S. U.; Beales, P. A.; Salonen, A.; Sawyer, L. J. Phys.: Condens. Matter 2000, 12, L569.

(18) Taratuta, V. G.; Holschbach, A.; Thurston, G. M.; Blankenschtein, D.; Benedek, G. B. J. Phys. Chem. 1990, 94, 2140.

(19) Tanaka, S.; Yamamoto, M.; Ito, K.; Hayakawa, R.; Ataka, M. Phys. Rev. E 1997, 56, R67.

(20) Drenth, J.; Haas, C. Acta Crystallogr. 1998, D54, 867; J. Phys. Chem. B 1998, 102, 4226; J. Cryst. Growth 1999, 196, 388.

(21) Rosenbaum, D. F.; Kulkarni, A.; Ramakrishnan, S.; Zukoski, C. F. J. Chem. Phys. 1999, 111, 9882.

(22) Gripon, C.; Legrand, L.; Rosenman, I.; Boué, F.; Regnaut, C. J. Cryst. Growth 1998, 183, 258.

(23) Hansen, J.-P.; McDonald, I. R. Theory of Simple Liquids; Academic Press: San Diego, CA, 1986.

(24) Thiele, E. J. Chem. Phys. 1963, 39, 474.

(25) Taniguchi, T.; Kawakatsu, T.; Kawasaki, K. In Slow Dynamics in Condensed Matter; Kawasaki, K., Kawakatsu, T., Tokuyama, M., Eds.; AIP Conference Proceedings 256; American Institute of Physics: New York, 1992; p 503.

(26) Sear, R. P. Eur. Phys. J. B 1998, 1, 313.

(27) Lapasin, R.; Pricl, S. Rheology of Industrial Polysaccharides: Theory and Applications; Blackie Academic: London, 1995.

(28) Tuinier, R.; Zoon, P.; Olieman, C.; Cohen Stuart, M. A.; Fleer, G. J.; De Kruif, C. G. Biopolymers 1999, 49, 1.

(29) Koning, M. M. G.; van Eendenburg, J.; de Bruijne, D. W. In Food Colloids and Polymers; Dickinson, E., Walstra, P., Eds.; Royal Society of Chemistry: Cambridge, U.K., 1993; p 103.

(30) Niimura, N.; Minezaki, Y.; Tanaka, I.; Fujiwara, S.; Ataka, M. J. Cryst. Growth 1999, 200, 265.

(31) Holt, C.; Timmins, P. A.; Errington, N.; Leaver, J. Eur. J. Biochem. 1998, 252, 73 .

(32) Odijk, T. Macromolecules 1996, 29, 1842.

BM0255544 